\title{
Guía didáctica de autoestima para el desarrollo social en preescolares
}

\author{
Byron Enrique Mora Avilez \\ byrforce1@hotmail.com \\ Universidad Estatal de Bolívar \\ Lincoln José Torres Valencia \\ lincoln.torresvalencia@yahoo.com \\ Universidad Estatal de Bolívar \\ Carlos Alfredo Gruezo González \\ cgruezo@ueb.edu.ec \\ Universidad Estatal de Bolívar \\ Andrea Alejandra Cevallos Goyes \\ acevallos@ueb.edu.ec \\ Universidad Estatal de Bolívar
}

\section{Línea de Investigación}

Diversidad del aprendizaje - enseñanza en el nivel inicial y preparatoria

\section{RESUMEN}

El presente artículo tiene como objetivo principal desarrollar y aplicar la guía didáctica de autoestima para determinar el desarrollo social, específicamente este estudio se lo realizó con los niños y niñas de primer año de Educación General Básica de la Escuela "Manuel de Echeandía" de la parroquia Veintimilla, cantón Guaranda, periodo lectivo 2017-2018. La guía didáctica resulta ser un documento de orientación técnica con herramientas y métodos didácticos que complementan el aprendizaje, mejorando la interacción pedagógica y la adquisición de competencias de autoestima para el desarrollo social de niños y niñas. La autoestima es un elemento básico y esencial para el desarrollo integral del ser humano, es una cualidad imprescindible para el éxito de las relaciones sociales, el logro de objetivos personales y académicos. El desarrollo social de los niños y niñas se desenvuelve en gran parte en la edad escolar y depende de los contextos en los que se desenvuelve con mayor frecuencia siendo estas la familia y el Centro Educativo. 
Es por ello que desde el Centro Educativo se debe guiar al niño a través de estrategias pedagógicas de autoestima por lo cual la presente guía didáctica implica diversas actividades estratégicas distribuidas en cinco bloques que son esenciales para mejorar el desarrollo social de los niños y niñas: nivel de comportamiento disruptivo, interacción, cooperación, aceptación social y nivel de apego. Dicha guía didáctica ha sido aplicada y evaluada la cual arroja los siguientes resultados: el nivel de comportamiento disruptivo pre se encontraba en 2,01 y post en 1,41 sobre 3, por lo que se puede constatar que existe una disminución de 0,60 lo que es equivalente a un 20\%. El nivel de interacción pre se encontraba en 1,62 y post en 2,02 sobre 3, por lo que se puede constatar que existe un mejoramiento de 0,41 lo que es equivalente a un 13,54\%. El nivel de cooperación pre se encontraba en 1,49 y post en 2,00 sobre 3, por lo que se puede constatar que existe un mejoramiento de 0,51 lo que es equivalente a un 16,97\%. El nivel de aceptación social pre se encontraba en 1,47 y post en 1,95 sobre 3 , por lo que se puede constatar que existe un mejoramiento de 0,48 lo que es equivalente a un 15,96\%. El nivel de apego pre se encontraba en 2,14 y post en 1,52 sobre 3, por lo que se puede constatar que existe una disminución de 0,62 lo que es equivalente a un 20,61\%. Previa al análisis de los resultados se concluyó que la guía didáctica de autoestima propuesta influye significativamente en el desarrollo social de los niños y niñas.

Palabras claves: guía didáctica; autoestima; desarrollo social; comportamiento disruptivo; interacción; cooperación; aceptación social y apego.

Artículo recibido: 05 octubre. 2021 Aceptado para publicación: 02 noviembre 2021 Correspondencia: byrforce1@ @hotmail.com Conflictos de Interés: Ninguna que declarar 


\section{Teaching guide of self-esteem for social development in preschool}

\section{ABSTRACT}

The main objective of this article is to develop and apply the didactic self-esteem guide to determine social development, specifically this study was carried out with the firstyear children of General Basic Education of the "Manuel de Echeandía" School of the parish Twenty-first, Canton Guaranda, school period 2017-2018. The didactic guide turns out to be a document of technical orientation with tools and didactic methods that complement the learning, improving the pedagogical interaction and the acquisition of self-esteem competitions for the social development of boys and girls.

Self-esteem is a basic and essential element for the integral development of the human being, it is an essential quality for the success of social relations, the achievement of personal and academic goals. The social development of children develops largely in school age and depends on the contexts in which it develops most frequently, these being the family and the Educational Center. That is why from the Educational Center should guide the child through self-esteem pedagogical strategies so the present didactic guide involves various strategic activities distributed in five blocks that are essential to improve the social development of children: level of disruptive behavior, interaction, cooperation, social acceptance and level of attachment. This didactic guide has been applied and evaluated which yields the following results: the level of disruptive behavior pre was in 2.01 and post in 1.41 over 3 , so it can be seen that there is a decrease of 0.60 which is equivalent to $20 \%$. The pre-interaction level was found at 1.62 and post at 2.02 on 3 , so it can be seen that there is an improvement of 0.41 , which is equivalent to $13.54 \%$. The pre-cooperation level was found at 1.49 and post at 2.00 out of 3 , so it can be seen that there is an improvement of 0.51 , which is equivalent to $16.97 \%$. The level of social acceptance pre was 1.47 and post in 1.95 out of 3 , so it can be seen that there is an improvement of 0.48 which is equivalent to $15.96 \%$. The level of pre attachment was found at 2.14 and post at 1.52 out of 3 , so it can be seen that there is a decrease of 0.62 , which is equivalent to $20.61 \%$. Prior to the analysis of the results, it was concluded that the proposed self-esteem didactic guide significantly influences the social development of children.

Keywords: teaching guide; self-esteem; social development; disruptive behavior; interaction; cooperation; social acceptance and attachment. 
Guía didáctica de...

\section{INTRODUCCIÓN}

El presente artículo "guía didáctica de autoestima para el desarrollo social en preescolares", en la que se trabajó específicamente con los niños y niñas de primer año de Educación General Básica de la escuela "Manuel de Echeandía” de la Parroquia Veintimilla, Cantón Guaranda, Periodo Lectivo 2017-2018, tiene como objetivo principal desarrollar y aplicar la guía didáctica de autoestima previo conocimiento de la realidad en la que se encontraba. Según (Kostelnik, Phipps Whiren, Soderman, \& Gregory, 2009) El desarrollo social es un estudio realizado para fomentar el desarrollo de habilidades sociales, considerando el amor propio y motivaciones acerca de lo que hacen, adquiriendo seguridad y confianza para una mejor convivencia. En la cual una de las variables más significativas para la adaptación social y el éxito en la vida es tener una autoestima positiva, es decir, saberse y sentirse competente en diferentes aspectos.

Según (Acosta Padrón \& Hernandez, La autoestima en la educación, 2008) la autoestima se basa en un carácter social y desarrollador que proveen de una extraordinaria significación para la educación de niños, adolescentes, jóvenes y adultos.

Según (UNICEF, 2015) los niños en la edad de 4 a 5 años se encuentran en una etapa en la cual intentan definir su personalidad, tomando diferentes modelos ya que se relacionan en distintos ámbitos: hogar, centro educativo, comunidad. El trabajo se centró en cinco variables, tomando como base la metodología de (Aguiar Sierra, 2010) que son los que permiten evaluar el desarrollo social de los niños y niñas los cuales son: comportamiento disruptivo, interacción, cooperación, aceptación social y apego. Se utilizó un estudio longitudinal ya que se realizó en dos etapas de tiempo; el primero un estudio pre con la aplicación de la ficha de observación que dio a conocer el estado actual del desarrollo social con respecto al autoestima y un estudio post después de la aplicación de la guía didáctica de autoestima para conocer su influencia en el desarrollo social de los niños y niñas. En la cual la ficha de observación aplicada permitió constatar que la guía didáctica de autoestima influyó significativamente en el mejoramiento de las relaciones sociales de los niños y niñas de primer año de Educación General Básica de la Escuela "Manuel de Echeandía”, específicamente el nivel de comportamiento disruptivo disminuyó un 20\%, la interacción mejoró un 13,54\%, la cooperación 16,97\%, la aceptación social un 15,96\% y el nivel de apego disminuyó en un $20 \%$. 


\section{METODOLOGÍA}

El tipo de estudio utilizado, según el objeto de estudio, fue investigación experimental por lo cual se realizó un estudio pre y post de la aplicación de la guía didáctica, según el marco en que tiene lugar fue investigación de campo dado que la información obtenida se desarrolló en el lugar de los hechos donde se encuentra el objeto del estudio, según la fuente de investigación se utilizó la investigación bibliográfica por lo cual el proceso investigativo en las cuales se sustenta y apegada la parte teórica de este documento se sustenta en libros, revistas, publicaciones, etc. según el método a utilizar fue método inductivo esto se debe a que utiliza una estrategia de razonamiento que parte de premisas particulares para generar conclusiones generales, se apoya en observaciones específica, también se utiliza el estudio cuantitativo porque se utiliza la ficha de observación de la cual se obtiene información basada en la observación de comportamientos naturales de los niños y niñas.

\section{Diseño de la investigación}

El diseño de la investigación aplicada es experimental esto se debe a que el investigador emplea una guía didáctica de autoestima para determinar la influencia del desarrollo social, según su ubicación temporal se utilizó el diseño longitudinal porque se obtuvo los datos en dos momentos (estudio pre y post de las variables).

\section{Población y muestra}

La población se constituye por los estudiantes de primer año de Educación General Básica Escuela "Manuel de Echeandía" de la parroquia Veintimilla, cantón Guaranda, los cuales son 33 estudiantes, dado que la población se considera pequeña y se puede trabajar con todos los estudiantes no se tomó una muestra, por lo cual se trabajó con el 100\% de la población.

\section{Técnicas e instrumentos para recolección de datos}

\section{Técnica}

La técnica que se utilizó para la recolección de datos es la observación, esta técnica facilitó valorar la incidencia de la aplicación de la guía de autoestima la misma que está conformada por cinco indicadores relacionados a los problemas y objetivos específicos.

\section{Instrumento}

El instrumento que se utilizó es la ficha de observación la misma que nos permitió realizar la recolección de la información de los cinco indicadores relacionados a los problemas y 
objetivos específicos.

\section{Procedimiento}

Los procedimientos realizados durante la investigación, se detallan a continuación: analizar el desarrollo social en los niños(as) del primer año de Educación Básica de la Escuela "Manuel de Echeandía", desarrollo ficha de observación, aplicación de la ficha de observación, tabulación de la información y obtención de resultados preliminares, desarrollo de la guía didáctica, etapa de pruebas a la población, aplicar la misma ficha de observación estudio post, tabulación de la información, estudio estadístico en el sistema SPSS, determinación de resultados para la prueba de hipótesis, resultados y conclusiones.

\section{Método estadístico}

Para el desarrollo del presente artículo se realizó la prueba estadística no paramétrica mediante el software estadístico SPSS, ya que los datos de los indicadores no cumplieron con la homogeneidad de las varianzas y la prueba de normalidad, por lo cual para la verificación de la hipótesis se realizó la prueba de rangos con signo de Wilcoxon con un nivel de confianza del 95\%, y un error del 5\% con una población de 33 estudiantes.

\section{RESULTADOS}

\section{Hipótesis}

- H0: La guía didáctica de autoestima no influye en el mejoramiento del desarrollo social de los niños y niñas de primer año de Educación General Básica de la Escuela "Manuel de Echeandía" de la parroquia Veintimilla, cantón Guaranda, periodo lectivo 2017-2018

- H1: La guía didáctica de autoestima influye en el mejoramiento del desarrollo social de los niños y niñas de primer año de Educación General Básica de la Escuela “Manuel de Echeandía" de la parroquia Veintimilla, cantón Guaranda, periodo lectivo 2017-2018.

\section{Los resultados obtenidos son:}

\section{Análisis de los indicadores}

\section{- Nivel de comportamiento disruptivo}

Posterior a la aplicación de las respectivas pruebas estadísticas, en la Tabla $1 \boldsymbol{y}$ Gráfico 1 se puede observar el nivel de comportamiento disruptivo obtenido según la guía de observación pre y post. 
Tabla 1. Nivel de Comportamiento disruptivo

\begin{tabular}{|l|c|}
\hline \multicolumn{1}{|c|}{ Indicador } & Nivel \\
\hline Pre nivel de comportamiento disruptivo & 2,01 \\
\hline Post nivel de comportamiento disruptivo & 1,41 \\
\hline
\end{tabular}

Fuente: Guía de observación

Elaborado por: Byron Enrique Mora Avilez

Gráfico 1. Nivel de Comportamiento disruptivo

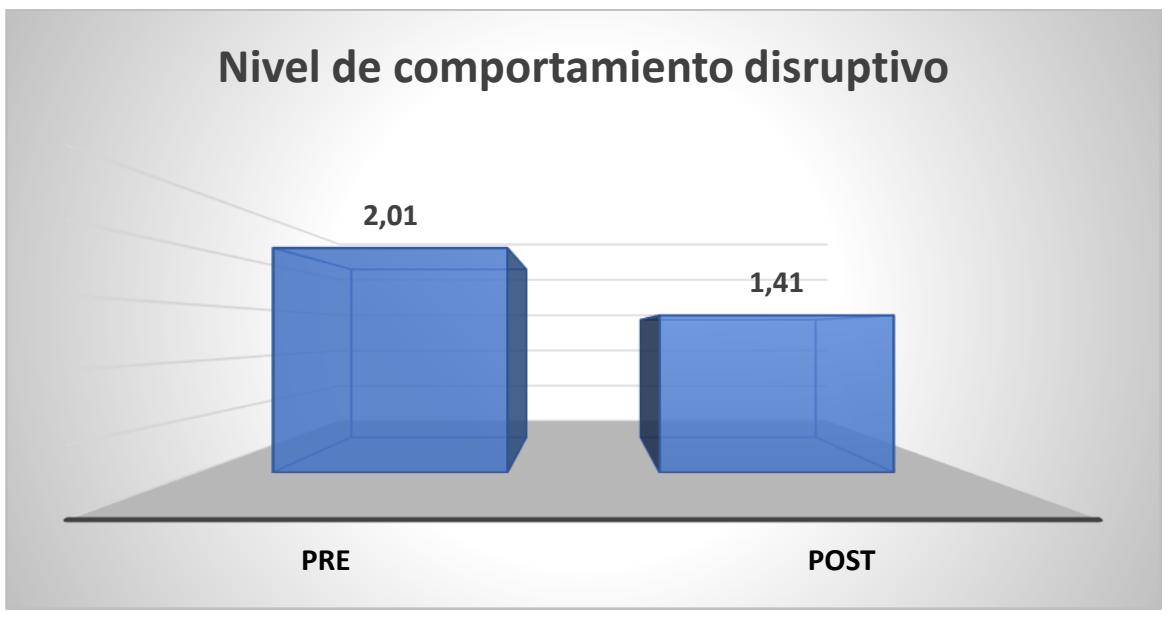

Fuente: Tabla 1. Nivel de Comportamiento disruptivo

Elaborado por: Byron Enrique Mora Avilez

Según la guía de observación aplicada el comportamiento disruptivo antes de la ejecución de la guía didáctica se encontraba en el nivel 2,01 sobre 3, posterior a la ejecución de la guía didáctica se reduce al nivel 1,41 sobre 3 , por lo cual podemos constatar la influencia positiva de la guía didáctica ya que ha contribuido para reducir los comportamientos inadecuados en los niños y niñas del primer año de Educación General Básica de la Escuela "Manuel de Echeandía".

\section{- Nivel de interacción}

Posterior a la realización de las respectivas pruebas estadísticas en la Tabla 2 y Gráfico 2 se puede observar el nivel de interacción obtenido según la guía de observación pre y post. 
Tabla 2. Nivel de Interacción

\begin{tabular}{|l|c|}
\hline Indicador & Nivel \\
\hline Pre nivel de Interacción & 1,62 \\
\hline Post nivel de Interacción & 2,02 \\
\hline
\end{tabular}

Fuente: Guía de observación

Elaborado por: Byron Enrique Mora Avilez

\section{Gráfico 2. Nivel de Interacción}

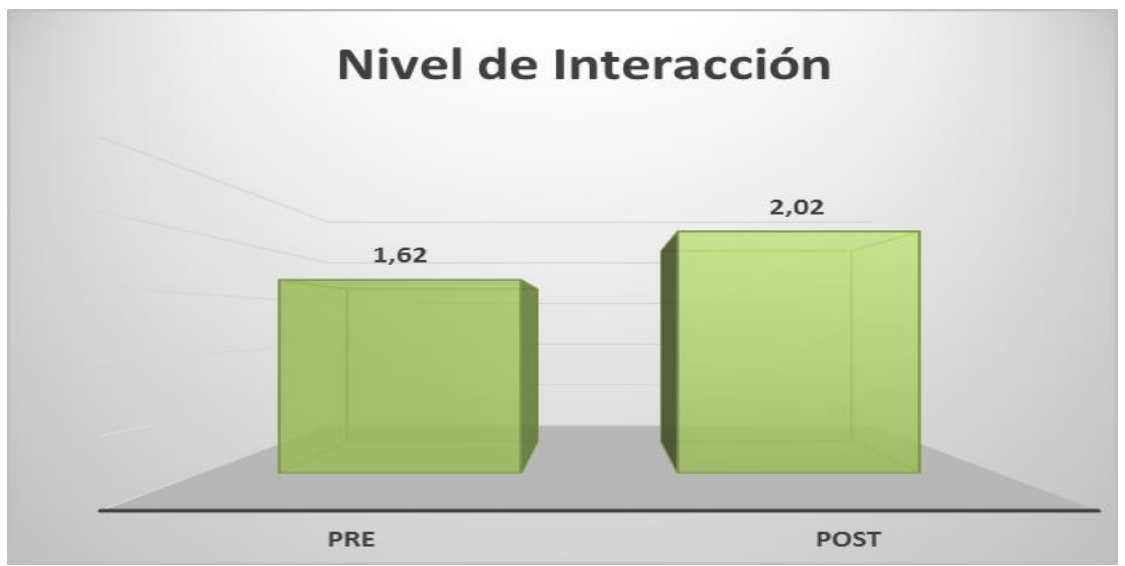

Fuente: Tabla 2. Nivel de Interacción

Elaborado por: Byron Enrique Mora Avilez

Según la guía de observación aplicada en lo referente a la interacción con los demás según el estudio pre o antes de la ejecución de la guía didáctica se encontraba en el nivel 1,62 sobre 3, posterior a la ejecución de la guía didáctica sube al nivel 2,02 sobre 3, por lo que podemos constatar la influencia positiva de la guía didáctica ya que ha contribuido al mejoramiento de la interacción con los demás de los niños y niñas del primer año de Educación General Básica de la Escuela "Manuel de Echeandía".

\section{Nivel de cooperación}

Posterior a la realización de las respectivas pruebas estadísticas en la Tabla 3 y Gráfico 3 se puede observar el nivel de cooperación obtenido según la guía de observación pre y post. 
Tabla 3. Nivel de Cooperación

\begin{tabular}{|r|c|}
\hline Indicador & Nivel \\
\hline Pre nivel de Cooperación & 1,49 \\
\hline Post nivel de Cooperación & 2,00 \\
\hline
\end{tabular}

Fuente: Guía de observación

Elaborado por: Byron Enrique Mora Avilez

\section{Gráfico 3. Nivel de Cooperación}

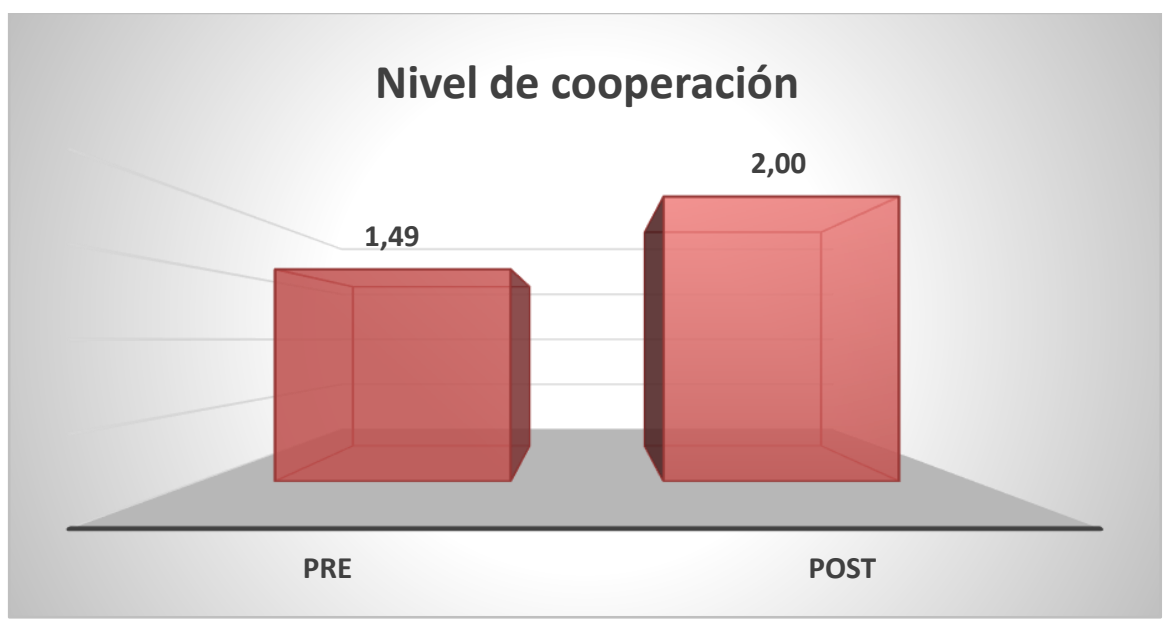

Fuente: Tabla 3. Nivel de Cooperación

Elaborado por: Byron Enrique Mora Avilez

El nivel de cooperación según la guía de observación aplicada pre o antes de la ejecución de la guía didáctica se encontraba en el nivel 1,49 sobre 3, luego de la ejecución de la guía didáctica asciende al nivel 2,00 sobre 3, por lo que podemos constatar una variación de mejora de la cooperación y colaboración con los demás.

\section{Nivel de aceptación social}

Posterior a la realización de las respectivas pruebas estadísticas en la Tabla 4 y Gráfico 4 se puede observar el nivel de Aceptación social obtenido según la guía de observación pre y post. 
Tabla 4. Nivel de Aceptación social

\begin{tabular}{|l|c|}
\hline \multicolumn{1}{|c|}{ Indicador } & Nivel \\
\hline Pre nivel de Aceptación Social & 1,47 \\
\hline Post nivel de Aceptación Social & 1,95 \\
\hline
\end{tabular}

Fuente: Guía de observación

Elaborado por: Byron Enrique Mora Avilez

Gráfico 4. Nivel de Aceptación social

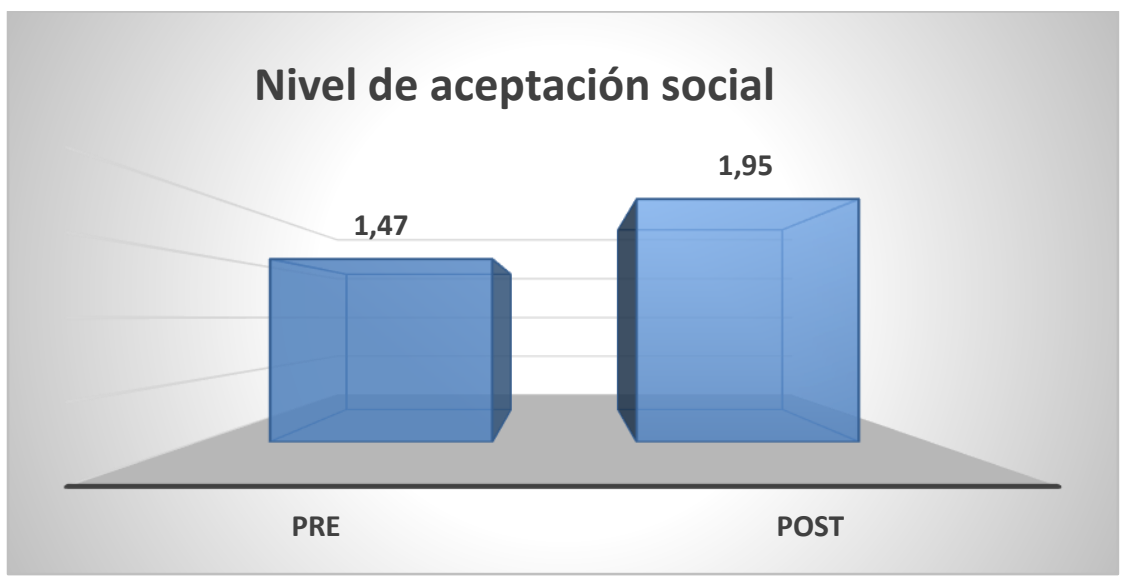

Fuente: Tabla 4. Nivel de Aceptación social

Elaborado por: Byron Enrique Mora Avilez

El nivel de aceptación social según la guía de observación aplicada pre o antes de la ejecución de la guía didáctica se encontraba en el nivel 1,47 sobre 3, luego de la ejecución de la guía didáctica asciende al nivel 1,95 sobre 3 , por lo que podemos constatar una variación de mejora de la aceptación social entre niños y niñas de primer año de Educación General Básica de la Escuela "Manuel de Echeandía" de la parroquia Veintimilla, cantón Guaranda, periodo lectivo 2017-2018.

\section{Nivel de apego}

Posterior a la realización de las respectivas pruebas estadísticas en la Tabla $5 \mathrm{y}$

Gráfico 5 se puede observar el nivel de apego obtenido según la guía de observación pre y post. 
Tabla 5. Nivel Apego

\begin{tabular}{|l|c|}
\hline \multicolumn{1}{|c|}{ Indicador } & Nivel \\
\hline Pre nivel de Apego & 2,14 \\
\hline Post nivel de Apego & 1,52 \\
\hline
\end{tabular}

Fuente: Guía de observación

Elaborado por: Byron Enrique Mora Avilez

Gráfico 5. Nivel Apego

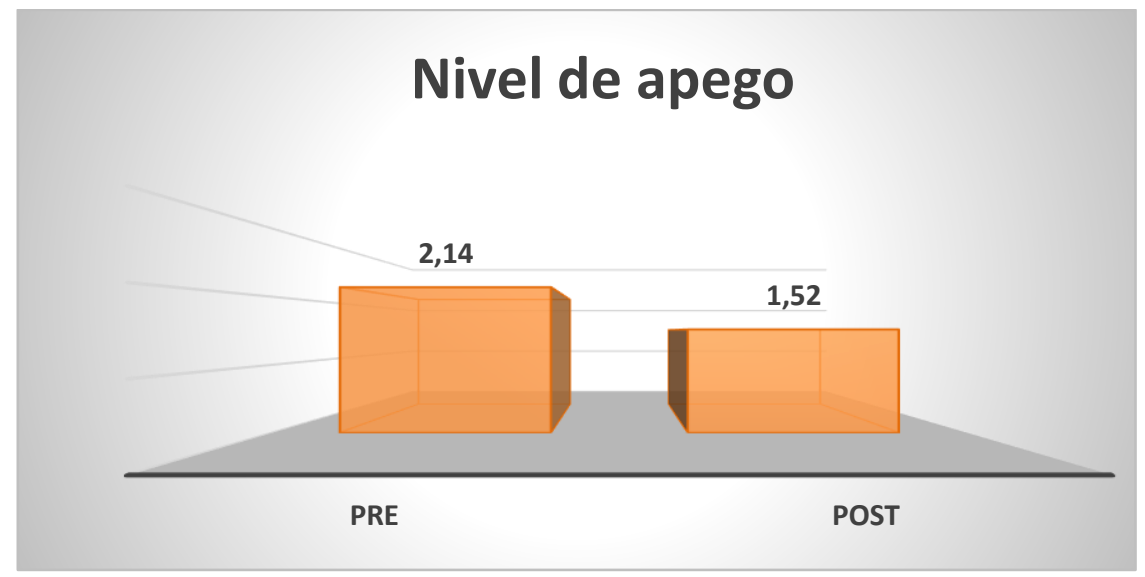

Fuente:

Tabla 5. Nivel Apego

Elaborado por: Byron Enrique Mora Avilez

El nivel apego según la guía de observación aplicada antes de la ejecución de la guía didáctica se encontraba en el nivel 2,14 sobre 3, luego de la ejecución de la guía didáctica se reduce al nivel 1,52 sobre 3, por lo que podemos verificar que el apego a los padres se ha reducido, de esta manera contribuido al desarrollo social de los niños y niñas primer año de Educación General Básica de la Escuela "Manuel de Echeandía" de la parroquia Veintimilla, cantón Guaranda, periodo lectivo 2017-2018.

\section{Datos consolidados}

Luego de realizar el análisis de cada uno de los indicadores se procedió a consolidar los datos para lo cual se aplica la Ecuación 1 para determinar el promedio total de los niveles por indicador. 
Tabla 6. Datos consolidados

\begin{tabular}{|l|c|c|c|c|c|c|}
\hline \multicolumn{1}{|c|}{ DATOS CONSOLIDADOS } \\
\hline $\begin{array}{l}\text { Indicadores } \\
\text { Nivel de comportamiento } \\
\text { disruptivo }\end{array}$ & 2,01 & 66,87 & 1,41 & 46,87 & 0,60 & 20,00 \\
\hline Interacción & 1,62 & 53,94 & 2,02 & 67,47 & 0,41 & 13,54 \\
\hline Cooperación & 1,49 & 49,70 & 2,00 & 66,67 & 0,51 & 16,97 \\
\hline Aceptación social & 1,47 & 48,89 & 1,95 & 64,85 & 0,48 & 15,96 \\
\hline Nivel de apego & 2,14 & 71,31 & 1,52 & 50,71 & 0,62 & 20,61 \\
\hline
\end{tabular}

Fuente: Guía de observación

Elaborado por: Byron Enrique Mora Avilez

En la Tabla 7 se detalla el nivel de desarrollo social de acuerdo a calificativos de los niveles de los indicadores estos calificativos se detallan en la ¡Error! No se encuentra el origen de la referencia..

Tabla 7. Datos consolidados según calificativos del nivel

\begin{tabular}{|l|c|c|}
\hline \multicolumn{1}{|c|}{ DATOS CONSOLIDADOS SEGÚN CALIFICATIVOS DEL NIVEL } \\
\hline \multicolumn{1}{|c|}{ Indicadores } & Guía pre & Guía post \\
\hline Nivel de comportamiento disruptivo & Alto & Medio \\
\hline Interacción & Medio & Alto \\
\hline Cooperación & Medio & Medio \\
\hline Aceptación social & Medio & Medio \\
\hline Nivel de apego & Alto & Medio \\
\hline
\end{tabular}

Fuente: Guía de observación

Elaborado por: Byron Enrique Mora Avilez 
Gráfico 6. Porcentaje de variación de los indicadores

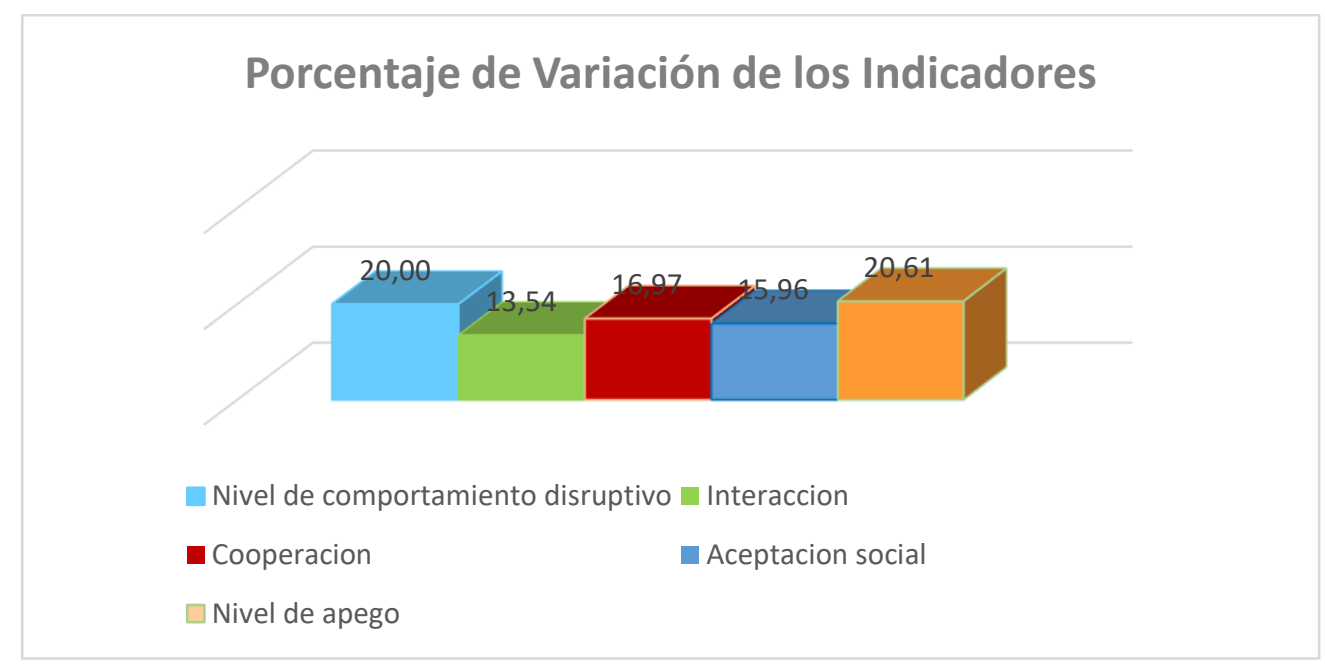

Fuente: Tabla 6. Datos consolidados

Elaborado por: Byron Enrique Mora Avilez

Posteríos a la aplicación de la ficha de observación aplicada pre y post se determinó la variación de los indicadores de desarrollo social de la siguiente manera: el nivel de comportamiento disruptivo disminuyó 0,60 lo que es equivalente a un $20 \%$, el nivel de interacción mejoró 0,41 lo que es equivalente a un 13,54\%, el nivel de cooperación mejoró 0,51 lo que es equivalente a un $16,97 \%$, el nivel de aceptación social mejoró 0,48 lo que es equivalente a un $15,96 \%$, el nivel de apego disminuyo 0,62 lo que es equivalente a un $20,61 \%$. Como se puede constatar los indicadores han tenido variaciones positivas que contribuyen al mejoramiento del desarrollo social.

\section{Comprobación de la hipótesis}

Luego de realizar un análisis de los indicadores muy detalladamente se concluyó que: la guía didáctica de autoestima influye en el mejoramiento del desarrollo social de los niños y niñas de primer año de Educación General Básica de la Escuela "Manuel de Echeandía" de la parroquia Veintimilla, cantón Guaranda, periodo lectivo 2017-2018. Por lo cual se rechazó la hipótesis $\mathrm{H} 0$ o hipótesis nula y se aceptó la hipótesis H1 o del investigador.

\section{Análisis final de los resultados}

Los factores determinantes de autoestima que incidieron en el desarrollo social de los niños y niñas de primer año de Educación General Básica de la Escuela "Manuel de Echeandía" de la parroquia Veintimilla, cantón Guaranda, periodo lectivo 2017-2018 fueron: comportamiento disruptivo, interacción, cooperación, aceptación social y apego. 
Posterior a la culminación de la aplicación de la guía didáctica, se determinó la influencia significativa de la guía didáctica de autoestima en las relaciones sociales de los niños y niñas en la cual se llegó a constatar que el nivel de comportamiento disruptivo disminuyó un $20 \%$, la interacción mejoró un 13,54\%, la cooperación 16,97\%, la aceptación social un $15,96 \%$ y el nivel de apego disminuyó en un $20 \%$.

\section{DISCUSIÓN}

Frente al estudio de aplicación de una guía didáctica para el desarrollo social, nos encontramos en la importancia que tiene la autoestima. En un estudio reciente de (Sevilla Villegas, 2012) en el que realiza un análisis a tres centros educativos: Centro Infantil mi pequeño Merani, Centro Infantil Función Judicial, Escuela de ciudad de Cuenca a niños comprendidos de 4 a 6 años se determinó que el 65\% de los niños mencionan que se burlan de ellos, esto se relaciona principalmente con el nivel comportamiento disruptivo de la presente investigación. Como podemos verificar existen factores que inciden negativamente en el desarrollo social de los niños y niñas, por lo cual la aplicación de la presente guía didáctica sería un aporte para la disminución del nivel de comportamiento disruptivo, ya que en la presente investigación se determinó que luego de la aplicación de la guía didáctica existió una disminución de un $66,87 \%$ a un $46,87 \%$ por lo cual el porcentaje de variación existente es de un 20\%, esto se pude verificar en el Gráfico 6. En cuanto al nivel interacción según (Torres Morales, 2011), determinó que el 20\% de los niños participan activamente y el 11\% de los niños participan voluntariamente lo cual da un promedio de 15,5\% Por lo cual la aplicación de la presente guía didáctica sería un aporte para el mejoramiento del nivel de interacción, ya que en la presente investigación se determinó que luego de la aplicación de la guía didáctica existió una aumento significativa de un $53,94 \%$ al $67,47 \%$ por lo cual existe un porcentaje de variación de un $13,54 \%$, esto se pude verificar en el Gráfico 6. En cuanto al nivel cooperación según (Torres Morales, 2011), determinó que el 27\% de los niños coopera con el arreglo del aula y el $20 \%$ de los niños respeta el orden de intervención lo cual da un promedio de 23,5\% Por lo cual la aplicación de la presente guía didáctica sería un aporte para el mejoramiento del nivel de interacción, ya que en la presente investigación se determinó que luego de la aplicación de la guía didáctica existió una aumento significativa de un $49,70,94 \%$ al $66,67 \%$ por lo cual existe un porcentaje de variación de un $16,97 \%$. esto se pude verificar en el Gráfico 6. En cuanto al nivel aceptación social según (Sevilla 
Villegas, 2012) determinó que el 62\% de los niños encuestados responden que tienen pocos amigos, $63 \%$ de los niños responden que les gusta jugara con los niños lo cual da un promedio de $62,5 \%$ Por lo cual la aplicación de la presente guía didáctica fue un aporte para el mejoramiento del nivel de la aceptación social, ya que en la presente investigación se determinó que luego de la aplicación de la guía didáctica existió una aumento significativa de un $48,89 \%$ al $64,85 \%$ por lo cual existe un porcentaje de variación de un $15,96 \%$ esto se puede verificar en el Gráfico 6. En un estudio de (Torres Morales, 2011) en el que realiza un análisis a niños de 3 a 4 años se determinó que el $25 \%$ de los padres responden que los niños se aíslan frente a personas desconocidas, esto se relaciona principalmente con el indicador apego de la presente investigación. Como podemos verificar existen factores que inciden negativamente en el nivel de apego de los niños y niñas, por lo cual la aplicación de la presente guía didáctica fue un aporte para la disminución del nivel de apego, ya que en la presente investigación se determinó que luego de la aplicación de la guía didáctica existió una disminución de un 71,31\% a un $50,71 \%$ por lo cual el porcentaje de variación existente es de un $20,61 \%$ esto se puede verificar en el Gráfico 6.

\section{CONCLUSIONES}

- Para poder lograr un desarrollo social adecuado en los niños y niñas de primer año de Educación General Básica de la Escuela "Manuel de Echeandía" es imprescindible realizar un estudio pre que permite conocer el nivel de autoestima existente.

- Se determinó que los factores que incidieron en el desarrollo social en base a la autoestima fueron: comportamiento disruptivo, interacción, cooperación, aceptación social y apego, dentro de los cuales los factores que requieren mayor tiempo de acompañamiento e intervención de parte de los docente y representantes son: cooperación y aceptación social.

- La guía didáctica de autoestima influyó significativamente en el mejoramiento de las relaciones sociales de los niños y niñas de primer año de Educación General Básica de la Escuela "Manuel de Echeandía", específicamente el nivel de comportamiento disruptivo disminuyó un 20\%, la interacción mejoró un 13,54\%, la cooperación $16,97 \%$, la aceptación social un 15,96\% y el nivel de apego disminuyó en un $20 \%$. 
Guía didáctica de...

\section{REFERENCIAS BIBLIOGRAFICAS}

Acosta Padrón , R., \& Hernandéz, J. (2009). La autoestima en la educación. Arica: Universidad de Tarapacá.

Acosta Padrón, R., \& Hernandez, J. A. (2008). La autoestima en la educación. Revista Límite, 9-10.

Aguiar Sierra, R. (2010). A Social Development Assessment Scale. Revista electrónica de investigación educativa, 16-20.

Aguilar Feijoo, R. M. (2010). La guía didáctica, un material educativo para promover el aprendizaje autónomo. Evaluación y mejoramiento de su calidad en la modalidad abierta y a distancia de la UTPL. Loja: Universidad Técnica Particular de Loja.

Alcántara, J. (2008). Cómo educar la autoestima. Madrid: CEAC.S.A.

Alkon, A., Bernzwelg, J., Boyer, L., Calder, J., \& Dailey, L. (2006). Desarrollo social y emocional de los niños. California: California Childcare Health Program.

Arauco. (15 de 08 de 2011). Fundacion Arauco. Obtenido de ¿ cómo hacer guías didácticas ?:

www.fundacionarauco.cl/_file/file_3881_guías\%20didácticas.pdf

Arranz Beltrán, E. (2010). Juegos cooperativos y sin competición para la educación infantil. Madrid: Semillas de paz.

Branden, N. (2010). Cómo mejorar su autoestima. Barcelona: Paidós Ibérica.

De la Orden Hoz, A. (s.f.). Función y características de las guías didácticas.

Director de programas UNICEF house. (4 de Julio de 2009). unicef.org. Recuperado el 12 fe Febrero de 2018, de http://files.unicef.org/supply/Activity_Guide_Spanishv1pdf.pdf

EL DIARIO. (29 de Mayo de 2018). El Diario. Obtenido de el diario.es: http://www.eldiario.ec/noticias-manabi-ecuador/472953-la-autoestima-es-clavede-felicidad/

Escorcia, I. S., \& Mejía Perez, O. (2015). Autoestima y pedagogía. EDUCARE, 243-244. Escuela de formación e innovación. (2014). Elaboración de Guías Didácticas. Murcia: Universidad de Murcia.

Felix, V. (2005). Conceptualización del comportamiento disruptivo en niños y adolescentes. Valencia: Universidad de Valencia. 
Kostelnik, M., Phipps Whiren, A., Soderman, A., \& Gregory, K. (2009). El desarrollo social de los niños. México: Cengage Learning Editores.

Larrabure, P., \& Paolicchi, G. (2016). Relación de tipo de apego con el juego infantil en la institución educativa. Buenos Aires: Universidad de Buenos Aires.

Lorenzo García, A. (2009). La Guía Didáctica. BENED.

Mongay Fernández, C. (2009). Quimiometría. Valencia: Universidad de Valencia.

Naranjo Pereira, M. (2009). Autoestima: un factor relevante en la vida de la persona y tema esencial del proceso educativo. Actualidades investigativas en educación, 45.

Proeva. (16 de 01 de 2013). Universidad de la república Uruguay. Obtenido de Elemento constitutivo de la guía didáctica: https://eva.udelar.edu.uy/mod/page/view.php?id=77793

Riso, W. (2013). Guia práctica para mejorar la autoestima. Madrid: Phónesis.

Sánchez Bautista, B., \& Solís Villafuerte, J. (12 de Marzo de 2010). Autoestima en niños. Obtenido de Ángeles Psicólogos, A.C : http://eoepsabi.educa.aragon.es/descargas/F_Educacion_emocional/f_7_autoesti ma/f_7.2.Obras_padres_madres/2.2..Autoestima\%20en\%20ninos.pdf

Schaffer, R. (2009). Desarrollo social. Madrid: Siglo XXI.

Sevilla Villegas, D. (2012). Factores familiares y escolares que afectan lña autoestima de los niños de 4 a 6 años. Propuesta alternativa. Quito: Escuela Politécnica del Ejército.

Shelov, S., \& Remer, T. (2009). CARING FOR YOUR BABY AND YOUNG CHILD: BIRTH TO AGE 5. New York: Academia Americana de pediatría.

Sher, B. (2008). Juegos para mejorar la autoestima en los niños. México: Selector.

Silva Escorcia, I. (2015). Autoestima, adolescencia y pedagogía. Educare Electronic Journal, 244.

Subsecretaría de fundamentos educativos. (2016). Instructivo para la aplicación de la evaluación estudiantil. Quito: Ministerio de educación.

Torres Morales, M. (2011). La autoestima en relación a la interacción en el aula, de los niños y niñas de 3 a 4 años del Centro de desarrollo Infantil "Atahualpa" año lectivo 2010-2011. Quito: Universidad Central del Ecuador. 
UNICEF. (2015). El desarrollo de niños y niñas de 4 a 10 años. Santiago de Chile: La Mandrágora Ltda.

UNICEF. (15 de Mayo de 2016). UNICEF para cada niño. Obtenido de Desarrollo de la primera infancia: https://www.unicef.org/es/desarrollo-de-la-primera-infancia

Vásquez Dodero, I. (2014). Cómo promover la autoestima de nuestros hijos e hijas. Madrid: CEAPA.

Villarreal González, M. E., Sanchez Sosa, J. C., \& Veiga, F. (2011). Contextos de desarrollo, malestar psicológico, autoestima social y violencia escolar. Madrid: Colegio Oficial de Psicólogos de Madrid.

Zenteno Durán, M. (2017). La autoestima y cómo mejorarla. Revistas Bolivarianas, 43 44. 


\section{ANEXOS}

Ecuación 1. Formula de Promedio

$$
\mathrm{p}=\frac{1}{2} \sum_{i-1}^{n} a=\frac{a 1+a 2+a 3+\cdots . . a n}{n}
$$

Tabla 8. Escala de Evaluación para Educación Inicial y Preparatoria

\begin{tabular}{|c|c|c|}
\hline Calificación & Respuestas & Escala \\
\hline $\mathbf{3}$ & Adquirido & A \\
\hline $\mathbf{2}$ & En Proceso & EP \\
\hline $\mathbf{1}$ & Iniciado & I \\
\hline
\end{tabular}

Elaborado por: Byron Enrique Mora Avilez

Tabla 9. Nivel de Evaluación y su equivalencia a Escala

\begin{tabular}{|c|c|}
\hline Rango & Nivel \\
\hline $2,01-3$ & Alto \\
\hline $1,01-2$ & Medio \\
\hline $0-1$ & Bajo \\
\hline
\end{tabular}

Elaborado por: Byron Enrique Mora Avilez

Estas escalas de evaluación fueron basadas en (Subsecretaría de fundamentos educativos, 2016). 